\section{Single step multiple genotyping by MALDI-TOF mass spectrometry, for evaluation of minor histocompatibility antigens in patients submitted to allogeneic stem cell transplantation from HLA-matched related and unrelated donor}

Federica Cattina, ${ }^{1}$ Simona Bernardi, 1 Vilma Mantovani, ${ }^{2}$ Eleonora Toffoletti, ${ }^{3}$ Alessandra Santoro, ${ }^{4}$ Domenico Pastore, ${ }^{5}$ Bruno Martino, ${ }^{6}$ Giuseppe Console, ${ }^{7}$ Giovanni Martinelli, $^{8}$ Michele Malagola ${ }^{1}$

${ }^{1}$ Unit of Blood Disease and Stem Cell Transplantation, Department of Clinical and Experimental Sciences, University of Brescia, ASST Spedali Civili of Brescia; ${ }^{2}$ Center for Applied Biomedical Research (CRBA), St. Orsola-Malpighi University Hospital, Bologna; ${ }^{3}$ Division of Hematology and Bone Marrow Transplantation, Department of Experimental and Clinical Medical Sciences, Azienda OspedalieroUniversitaria di Udine; ${ }^{4}$ Division of Hematology and Bone Marrow Transplantation, Ospedali Riuniti Villa Sofia-Cervello, Palermo; ${ }^{5}$ Hematology and Bone Marrow Transplantation Unit, Department of Emergency and Organ Transplantation, University of Bari; ${ }^{6}$ Hematology Division, AO BMM, Reggio Calabria; ${ }^{7}$ Hematology and Bone Marrow Transplant Unit, AO BMM, Reggio Calabria; ${ }^{8}$ Department of Experimental, Diagnostic and Specialty Medicine, Hematology "L.eA. Seràgnoli”, University of Bologna, Italy

\footnotetext{
Abstract

The outcome of patients underwent to allogeneic stem cell transplantation (allo$\mathrm{SCT}$ ) is closely related to graft versus host disease (GvHD) and graft versus leukemia $(\mathrm{GvL})$ effects which can be mediated by mHAgs. 23 mHAgs have been identified and reported to be differently correlated with GVHD or GVL and the aim of this work was develop a method to genotype the mHAgs described so far. For this study we used MALDI-TOF iPLEX Gold Mass Array technology. We tested 46 donor/recipient matched pairs that underwent allo-SCT
}

because of Philadelphia positive $(\mathrm{Ph}+)$ chronic myeloid leukemia $(\mathrm{n}=29)$ or $\mathrm{Ph}+$ acute lymphoblastic leukemia $(n=17)$. Our data show that sibling pairs had a lesser number of mHAgs mismatches compared to MUD pairs. Notably, donor/recipient genomic mismatch on DPH1 was correlated with an increased risk of acute GvHD and LB-ADIR-1R mismatch on graft versus host direction was correlated with a better RFS with no increase of GvHD risk. Our work provides a simple, accurate and highly automatable method for mHAgs genotyping and suggest the role of mHAgs in addressing the immune reaction between donor and host.

\section{Introduction}

Allogeneic stem cell transplantation (allo-SCT) may be the only cure for patients affected by acute myeloid or lymphoid leukemia, or other hematological diseases such as lymphomas or multiple myeloma. ${ }^{1}$

The curative effects of allo-SCT are closely related to graft versus leukemia $(\mathrm{GvL})$. However the severity of the graft versus host disease (GvHD) may override the GvL benefit and worsen the outcome of allotransplanted patients. ${ }^{1-3}$ Despite a full major HLA antigens (MHAgs) compatibility, minor histocompatibility antigens (mHAgs) can also play a pivotal role in conditioning both GvL and GvHD response in HLA full-matched allo-SCT. Evidence from experimental and clinical studies on HLA-identical allo-SCT suggest that GvL and GvHD may be driven by donor T cell responses against disparate mHAgs. ${ }^{4-}$ 9 Indeed, mHAgs are polymorphic HLA-bound peptides derived from cellular proteins that can induce powerful alloreactive $\mathrm{T}$ cell responses. The mHAgs recipient-donor disparity may arise from a genomic variation in the coding region of the gene that leads to differences in the amino acid sequence of the homologous protein and, in most cases, it may depend on a nonsynonymous single nucleotide polymorphism (nsSNP) or on a deletion.7,10,11 Recent advances in the molecular identification of mHAgs have significantly expanded our knowledge to a total of 23 autosome-coded mHAgs and 10 Y-chromosome coded mHAgs, leading to an increased interest in the clinical application of mHAgs typing. Although several mHAgs, including Y-chromosome encoded mHAgs, are ubiquitously expressed, an increasing number of autosomal-encoded mHAgs is being identified as expressed exclusively by hematopoietic cells or by their malignant counterparts. ${ }^{12-21}$
Correspondence: Cattina Federica, Unit of Blood Disease and Stem Cell Transplantation, Department of Clinical and Experimental Sciences, University of Brescia, ASST Spedali Civili of Brescia, P.le Ospedali Civili 1, 25123 Brescia, Italy.

Tel.: +39.339.1624072 - Fax: +39030 3996021 . E-mail: cattina.federica@libero.it

Key words: Stem cell transplantation, HLA, minor Histocompatibility antigens.

Contributions: $\mathrm{FC}$ and $\mathrm{MM}$ designed the study; FC, SB and MM wrote the manuscript; FC, ET, AS, DP, BM, GC, MM and GM collected the samples; FC, SB, VM performed the laboratory analysis; all the Authors reviewed the manuscript before submission.

Conflict of interest: The authors declare no potential conflict of interest.

Funding: this work was supported in part by Banca di Credito Cooperativo di Pompiano e Franciacorta and Lions Club Bassa Bresciana Association.

Received for publication: 17 January 2017. Accepted for publication: 11 April 2017.

This work is licensed under a Creative Commons Attribution-NonCommercial 4.0 International License (CC BY-NC 4.0).

(C) Copyright F. Cattina et al., 2017

Licensee PAGEPress, Italy

Hematology Reports 2017; 9:7051

doi:10.4081/hr.2017.7051

About this, ACC-1, ACC-2 and HA-2 have been correlated with the beneficial GvL effects, while some mHAgs disparities, CD31, HA-5, HA-8 and UGT2B17, have been found to be involved in the induction of GvHD. ${ }^{8,22-28}$

The molecular identification of GvHDand GvL-associated mHAgs could allow the evaluation of the clinical impact of mHAgs mismatches and their specific $\mathrm{T}$ cell responses triggered by allo-SCT. Several studies in HLA-matched allo-SCT reported an association between mHAgs mismatches and the clinical outcome, ${ }^{29-33}$ but other studies have not confirmed these observations. $^{7,24}$

The heterogeneity of techniques suitable for mHAgs typing (SSP-PCR and Luminex) as well as the complexity of integrating mHAgs typing data and clinical information are likely the main reasons that do not facilitate the routinary evaluation of mHAgs in clinics. ${ }^{34-36}$ In our study, we set up a new method for mHAgs genotyping based on Matrix Assisted Laser Desorption Ionization Time-of-Flight (MALDI-TOF) 
mass spectrometry (MS) and we tested it in a training set of donor-recipient pairs with the aim to propose a simple and standardizable methodology able to overcome the limits of the conventional methods and to make mHAgs genotyping suitable for clinical application. ${ }^{37-38}$

\section{Materials and Methods}

\section{Patients and transplant procedures}

For this study, we tested the MALDITOF iPLEX Gold method on a cohort of Ph+ $\mathrm{CML}$ and $\mathrm{Ph}+\mathrm{ALL}$ patients who underwent allo-SCT at six Italian Centres from 1990 to 2011. To this purpose, we retrospectively selected 46 donor-recipient pairs fully HLA compatible for HLA-A, -B, -C, -DRB1 and -DQB1 alleles, according to SSP-PCR high resolution molecular methods. Out of the 46 selected cases, 29 were $\mathrm{Ph}+\mathrm{CML}$ and 17 were $\mathrm{Ph}+\mathrm{ALL}$ patients who underwent alloSCT by sibling (29 cases, 63\%) or MUD (17 cases, 37\%).

GvHD effects, either acute or chronic, were defined according to the Glucksberg scale and NHI criteria, respectively, and they were reported as cumulative incidence. Relapse free survival (RFS) was calculated using Kaplan-Meier method and it was assumed as an indicator of GVL effect. ${ }^{39-40}$ All patients provided informed consent according to the policy of each participating Centre. Patients and transplant features are reported in Table 1.

\section{mHAgs's biological characteristics and definitions}

The HLA matched donor-recipient pairs evaluated for this study were genotyped for a panel of 23 mHAgs (and causal SNPs). The biological characteristics of each mHAg (gene, locus, SNP reference number, nucleotide switch and HLA restriction) are detailed in Table 2. We specify that CD31 exists in two isoforms (CD31125 and CD31563) because it results from two different SNPs (rs668 and rs12953, respectively). We genotyped both SNPs, but we considered the two isoforms together during the analysis because of the strong linkage between the two SNPs. On the contrary, the SNP rs2289702 determine two different mHAgs, ACC-4 and ACC-5, according to the HLA molecule that present them.

For the purpose of this study, immunogenic mHAg difference was defined when within a given donor/recipient pair, only one individual had an immunogenic phenotype of a particular mHAg accompanied by the appropriate HLA restriction molecule. Genomic mHAg difference was identified when mHAg genotypes in donor and recipient were different, but phenotypically they were either the same or the mHAg immunogenic phenotype was not accompanied by the appropriate HLA restriction molecule. Both genomic and immunogenic mHAgs disparities were included in the analysis. This is due to an incomplete knowledge of mHAgs because the epitope-prediction strategy often makes it hard to confirm the immunogenicity of the predicted putative mHAgs and there is currently no controlled way of isolating mHAgs-specific $\mathrm{T}$ cells directed against mHAgs.

\section{mHAgs genotyping by MALDI-TOF iPLEX Gold technology}

For the purpose of our study, the genomic DNA (gDNA) was extracted using QIAamp DNA mini Kit (Qiagen) from peripheral blood mononuclear cells (PBMC) previously cryopreserved. The PBMC collection was performed before allo-SCT for patients and before stem cells harvest for donors. The purity of gDNA for each sample was determined by measuring the absorbance at 260 and $280 \mathrm{~nm}$, with the A260/A280 values being in the range of 1.51.9 , and the concentration of the gDNA was adjusted to $12 \mathrm{ng} / \mu \mathrm{L}$. A total of $30 \mathrm{ng}$ of gDNA was used for genotyping all SNPs.

MS MALDI-TOF iPlex Gold is able to

Table 1. Patients and HSCT characteristics.

Characteristic
Age, mean (range)
Male
Male-female sex mismatch
Matched sibling donor
Matched unrelated donor
$\mathrm{Ph}+\mathrm{CML}$
$\quad \mathrm{CP}$
$\mathrm{AP} / \mathrm{BP}$
$\mathrm{Ph}+\mathrm{ALL}$
$\quad$ st CR
2nd CR
Relapse

Stem cell source

Mobilized peripheral blood $\quad 24$

CD34 $\times 10^{6} / \mathrm{kg}$, median (range) $\quad 5.07 \quad 2.2-8$

CD3 $\times 10^{6} / \mathrm{kg}$, median (range) $\quad 162$

Bone marrow, n (\%) 19

CD34 $\times 10^{6} / \mathrm{kg}$, median (range) $\quad 3.2$

CD $3 \times 10^{6} / \mathrm{kg}$, median (range) $\quad 23.5 \quad 20-40$

Interval between diagnosis and SCT

$\begin{array}{lcc}\leq 1 \text { year } & 24 & 52 \\ >1 \text { year } & 17 & 37 \\ \text { Not available } & 5 & 11\end{array}$

Date of SCT

1990-1999 19

$\begin{array}{lll}2000-2012 & 27 & 59\end{array}$

\begin{tabular}{lcc} 
Conditioning regimens & & \\
Busulfan based & 27 & 59 \\
TBI based & 17 & 37 \\
Others & 2 & 4 \\
\hline
\end{tabular}

GvHD prophylaxis

$\begin{array}{lll}\text { Cyclosporine/MTX } & 46 & 100\end{array}$

$\begin{array}{ccc}\text { Gratwohl score } & & \\ 1 & 6 & 13 \\ 2 & 12 & 26 \\ 3 & 11 & 24 \\ 4 & 6 & 13 \\ 5 & 4 & 9 \\ \geq 6 & 1 & 2 \\ \text { ND } & 6 & 13\end{array}$

CP, chronic phase; AP/BP, accelerate phase/blastic phase; CR, complete remission; TBI, total body irradiation; MTX, methotrexate. 
discriminate the two variants of an SNP in a very efficient way, so it was considered suitable for the aim of the study. The MassARRAY Assay Design software was used to design 3 different multiplex reactions to investigate the 23 SNPs. Genotyping was performed using iPLEX Gold technology and MassARRAY high-throughput DNA analysis with matrix-assisted laser desorption/ionization time-of-flight (MALDI $\square$ TOF) MS [Agena Bioscience Inc., San Diego, CA], according to the manufacturer's protocol. ${ }^{41}$ Multiplex design and primer sequences are shown in Table 3 .

The multiple-genotyping assay was validated using intra- and extra-run controls. Firstly, a DNA sample (NA10859) from the CEPH (Centre d'Etude du Polymorphisme Humain CEPH, Paris, France) panel was genotyped simultaneously in every single run. Six mHAgs (ACC-1, ACC-2, ACC-6, HA-8, HB-1 and LB-ADIR-1R) were reported. Then, the genotype of each polymorphism was validated in 10 randomly selected samples by amplification with PCR and subsequent direct Sanger Sequencing (ABI Prism 3730, Applied Biosystems, Foster City, CA) as gold standard.

\section{Statistical analysis}

For continuous factors, the median and ranges were calculated. The $\chi^{2}$-test was used to compare differences in percentage, and Mann-Whitney U test was used to compare continuous values. The probability of GvHD (acute and chronic) was estimated as cumulative incidence. In GvHD analysis, competing risks were relapse or death before the onset of GvHD. Probabilities for RFS were calculated using the Kaplan-Meier method. ${ }^{42}$ RFS was calculated from the date of allo-SCT until the date of relapse or death, whichever occurred first. Death in remission was considered as a competing risk in the relapse analysis. Differences in RFS were evaluated by log-rank testing in univariate analysis. Multivariate analyses were performed using the Fine and Gray regression model. The Cox proportional hazard regression model was used for multivariate analyses of variables affecting RFS. The following patient- and transplantrelated variables were analyzed: CML or ALL diagnosis and type of bcr-abl transcript, immunogenic/genomic mHAgs mismatches between donor and recipient, patient age at SCT, type of donor, patient gender and sex mismatch between donor and recipient, graft source, time from diagnosis to HSCT, conditioning regimen, GvHD prophylaxis and development of GvHD. All P-values were 2 -sided and $\mathrm{P}<0.05$ was considered statistically significant. Each SNP was tested for departures from the Hardy-Weinberg equilibrium (HWE).

\section{Results \\ SNPs genotyping by MALDI-TOF iPLEX Gold technology}

The MALDI-TOF iPLEX Gold technology method was used on a training

Table 2. mHAgs biological features.

\begin{tabular}{|c|c|c|c|c|c|c|c|c|}
\hline mHAg & Gene & $\begin{array}{l}\text { Gene } \\
\text { locus }\end{array}$ & $\begin{array}{c}\text { Tissue } \\
\text { expressione }\end{array}$ & $\begin{array}{l}\text { SNP } \\
\text { reference }\end{array}$ & $\begin{array}{l}\text { Nucleotide } \\
\text { switch }\end{array}$ & Restriction & $\begin{array}{l}\text { A } \\
\text { Caucasian } \\
\text { frequence }\end{array}$ & Ref. \\
\hline ACC-1 & Bcl2Al & $15 \mathrm{q} 24.3$ & Hemopoietic & rs1138357 & $\mathrm{G} \rightarrow \mathrm{A}$ & $A^{*} 24$ & 25 & {$[3,28,32,26,16]$} \\
\hline ACC-2 & Bcl2 & $15 \mathrm{q} 24.3$ & Hemopoietic & rs3826007 & $\mathrm{G} \rightarrow \mathrm{A}$ & $\mathrm{B}^{*} 44$ & 17 & {$[3,28,32,26]$} \\
\hline ACC-4 & Catepsina $\mathrm{H}$ & $15 q 24-25$ & Hemopoietic & rs2289702 & $\mathrm{G} \rightarrow \mathrm{A}$ & $\mathrm{A}^{*} 33: 03$ & 0 & {$[6,28]$} \\
\hline ACC-5 & Catepsina $\mathrm{H}$ & $15 q 24-25$ & Hemopoietic & rs2289702 & $\mathrm{G} \rightarrow \mathrm{A}$ & $A^{*} 31: 01$ & 0 & {$[6,28]$} \\
\hline ACC-6 & HMSD & 18q21.33 & Hemopoietic & rs9945924 & $\mathrm{G} \rightarrow \mathrm{A}$ & $\mathrm{B}^{*} 44$ & 17 & [6] \\
\hline C19orf48 & C19orf48 & $19 q 13$ & Broad & rs3745526 & $\mathrm{A} \rightarrow \mathrm{T}$ & $A^{*} 02: 01$ & 46,5 & {$[6,19]$} \\
\hline CD31 & PECAM1 & $17 q 23$ & Broad & rs668 & $\mathrm{C} \rightarrow \mathrm{G}$ & $\mathrm{A}^{*} 02$ & 52 & [27] \\
\hline CD31 & PECAM1 & $17 q 23$ & Broad & rs 12953 & $\mathrm{~A} \rightarrow \mathrm{G} / \mathrm{T}$ & $\mathrm{A}^{*} 02$ & 52 & {$[7,29]$} \\
\hline CTL7A7 & PANE-1 & $22 q 13.2$ & Hemopoietic & rs5758511 & $\mathrm{C} \rightarrow \mathrm{T}$ & $\mathrm{A}^{*} 03$ & 20 & {$[18,28,32]$} \\
\hline DPHl & DPH1 & 17 & Broad & rs35394823 & $\mathrm{C} \rightarrow \mathrm{G}$ & B*5701 & 4 & [28] \\
\hline DRN-7 & SP110 & $2 q 37.1$ & Hemopoietic & rs 1365776 & $\mathrm{G} \rightarrow \mathrm{A}$ & $\mathrm{A}^{*} 03$ & 20 & {$[28,32]$} \\
\hline HA-1 & KIA A0223 & 19p 13.3 & Hemopoietic & rs 1801284 & $\mathrm{~A} \rightarrow \mathrm{G}$ & $\begin{array}{c}\mathrm{A}^{*} 02 \\
\mathrm{~A}^{*} 02: 06 \\
\mathrm{~B} * 40: 01\end{array}$ & $\begin{array}{c}52, \\
0 \\
0\end{array}$ & {$[32]$} \\
\hline HA-2 & MYOG 1 & 7p13-p11.2 & Hemopoietic & rs61739531 & $\mathrm{G} \rightarrow \mathrm{A}$ & $\mathrm{A}^{*} 02: 01$ & 46,5 & {$[3,7,22,28,32,26]$} \\
\hline HA-3 & LBC & $15 q 24-25$ & Broad & rs7162168 & $\mathrm{T} \rightarrow \mathrm{C}$ & $\mathrm{A}^{*} 01$ & 21 & {$[3,10,27,28,32]$} \\
\hline HA-8 & KIAA 0020 & 9 p24.2 & Broad & rs2173904 & $\mathrm{G} \rightarrow \mathrm{C}$ & $\mathrm{A}^{*} 02: 01$ & 46,5 & {$[3,7,10,15,28,32,25]$} \\
\hline HB-1 & HB-1 & $5 q 31.3$ & Hemopoietic & rs161557 & $\mathrm{C} \rightarrow \mathrm{T}$ & $\begin{array}{l}\text { B*44:02, } \\
\text { B*44:03 }\end{array}$ & $\begin{array}{l}7 \\
20\end{array}$ & {$[3,13,28,32,26]$} \\
\hline HEATR-1 & HEATR-1 & 1q43 & Broad & rs2275687 & $\mathrm{C} \rightarrow \mathrm{T}$ & $\mathrm{B}^{*} 08: 01$ & 10 & [26] \\
\hline LB-ADIR-1R & TOR3A & 1q25.2 & Hemopoietic & rs2296377 & $\mathrm{T} \rightarrow \mathrm{C}$ & $\mathrm{A}^{*} 02: 01$ & 46,5 & [28] \\
\hline LB-ECGF-1H & ECGF & 22q13.33 & Hemopoietic & rs112723255 & $\mathrm{C} \rightarrow \mathrm{T}$ & $\mathrm{B} * 07$ & 11 & [28] \\
\hline LB-LY75-1K & Ly75 & $2 q 24.2$ & Hemopoietic & rs12692566 & $\mathrm{T} \rightarrow \mathrm{G}$ & DRB1*13:01 & 11 & [20] \\
\hline LB-MTHFD1-1Q & MTHFD1 & 14 & Hemopoietic & rs2236225 & $\mathrm{G} \rightarrow \mathrm{A}$ & DRB1*03:01 & 14 & [20] \\
\hline LB-PTK2B-1T & PTK2B & 8 & Hemopoietic & rs751019 & $\mathrm{A} \rightarrow \mathrm{C}$ & DRB3*01:01 & nd & [20] \\
\hline P2RX7 & P2RX7 & 12 & Broad & rs7958311 & $\mathrm{A} \rightarrow \mathrm{C} / \mathrm{G} / \mathrm{T}$ & $\mathrm{DRB} 1 * 03$ & 16 & {$[26]$} \\
\hline UTA2-1 & C12orf75 & 12 & Broad & rs2166807 & $\mathrm{A} \rightarrow \mathrm{G}$ & $A^{*} 02$ & 52 & {$[3]$} \\
\hline
\end{tabular}


group of 46 donor/recipient pairs with the aim to evaluate the accuracy and reliability of the genotyping assay. A total of 2116 genotypes resulted out of a predicted total number of 2116 (92 samples for $23 \mathrm{SNPs}$ ) with a call rate of $100 \%$.

In order to evaluate the accuracy and reliability of the genotyping assay, two different approaches were adopted. Evaluation of method reproducibility was carried out by genotyping of the DNA number NA10859 during the Sequenom run. This standard DNA is released the genotype of only six (6 of $23,26 \%$; ACC-1, ACC-2, ACC-6, HA-8, HB-1 and LB-ADIR-1R) mHAgs. The concordance between the released data and our genotyping was $100 \%$. In the second stage, we validated the set of 10 randomly selected samples using conventional Sanger sequencing and also in this case we obtained a concordance of $100 \%$. The Hardy-Weinberg equilibrium (HWE) was satisfied for most SNPs on both populations (patients and donors). rs12692566 (mHAgs LB-LY751K) was the only SNP showing a significant difference as compared with the prediction under HWE assumptions. Since Hardy Weinberg disequilibrium can indicate genotyping errors or population stratification, LBLY751K was excluded from the statistical analysis (Table 4).
mHAgs mismatches, patients' clinical features and correlation with GvHD/GvL effects

The analysis of immunogenic mismatches showed that sibling pairs had a lesser number of mismatches compared to MUD pairs (median 1 vs. 3; t-test with $\mathrm{P}<0.003$ ). The evaluation of genomic mismatches point out that sibling pairs have higher identity than MUD pairs (t-test, $\mathrm{P}<0.0001)$. In fact, the median number of genomic differences was 8 (range $0-15$ ) in sibling pairs and 13 (range 11-17) in MUD pairs (t-test with $\mathrm{P}<0.05$ ). Only one sibling pair showed a perfect genomic mHAgs match.

We also tried to correlate if some mHAgs mismatches could be involved in GvHD development. DPH1 genomic mismatch resulted to be correlated with the risk of grade $\geq 2$ aGvHD development (multivariate analysis HR 2.2, $\mathrm{P}=0.04$, Table 5), while no mHAgs mismatches were found to be correlated with an increased risk of cGvHD (Table 5).

By these evidences, we investigated any correlation between mHAgs mismatches and RFS as a clinical surrogate of GvL effect. Despite some clinical factors affecting the RFS (i.e. the underlying disease, b3a2 transcript isoform and chronic GvHD development), in multivariate analysis we observed that only LB-ADIR-1R, with genomic mismatch on graft versus host direction (HR 0.3, P=0.03, Table 5) was positively correlated with a better RFS.

\section{Discussion}

The study aimed to set up a new laboratory assay for genotyping minor histocompatibility antigens which are thought to play a key role in the allo-immune responses in fully HLA-matched stem cell transplantations.

The MALDI-TOF iPLEX gold approach was used to overcome the limits of conventional methods, such as SSP-PCR and Luminex, and to make mHAgs genotyping analysis suitable for clinical application. PCR-SSP and Luminex are commonly used for HLA typing, but both methods have several limitations. Complex primer design and identification of the annealing temperature are critical for the PCR-SSP test; while biotinylated DNA probes, beads and streptavidin-phycoerythrin binding are critical steps for Luminex. ${ }^{34,35}$ MALDI-TOF was used effectively for KIR (killer-cell immunoglobulin-like receptor) and platelet antigens genotyping and, due to the expected advantages in terms of rapidity, simplicity and high throughput capability, it was

Table 3. Primers using for MALDI-TOF assays are listed; PCR primers tags are in bold, no-template bases are reported in lower case letters.

\begin{tabular}{|c|c|c|c|c|c|}
\hline Multiplex & mHAg & SNP & $\begin{array}{l}\text { Amplification Prim } \\
\text { Forward }\end{array}$ & Reverse & Extension Primer \\
\hline 1 & $\begin{array}{l}\text { ACC-1 } \\
\text { ACC-2 } \\
\text { ACC-6 } \\
\text { C19orf48 } \\
\text { DRN7 } \\
\text { HA-1 } \\
\text { HA-2 } \\
\text { HA-8 } \\
\text { LB-ADIR-1R } \\
\text { LB-ECGF1 } \\
\text { LB-LY751K } \\
\text { LB-MTHFD1 } \\
\text { P2RX7 } \\
\text { UTA2-1 }\end{array}$ & $\begin{array}{l}\text { rs } 1138357 \\
\text { rs3826007 } \\
\text { rs } 9945924 \\
\text { rs3745526 } \\
\text { rs1365776 } \\
\text { rs1801284 } \\
\text { rs61739531 } \\
\text { rs2173904 } \\
\text { rs2296377 } \\
\text { rs112723255 } \\
\text { rs12692566 } \\
\text { rs2236225 } \\
\text { rs } 7958311 \\
\text { rs2166807 }\end{array}$ & $\begin{array}{l}\text { ACGTTGGATGTTGGACCTGATCCAGGTTGT } \\
\text { ACGTTGGATGTGGTTACAATTCTTCCCCAG } \\
\text { ACGTTGGATGGAAGTCCAGCTCAACTGATA } \\
\text { ACGTTGGATGCACGCCTAGGCAGGAAACA } \\
\text { ACGTTGGATGCTTCCTCTTGTACTCTCATC } \\
\text { ACGTTGGATGGCCTTGAGAAACTTAAGGAG } \\
\text { ACGTTGGATGATGGCCTCAGGCCCATACAG } \\
\text { ACGTTGGATGGTTTGTTGCAGTCAGCAG } \\
\text { ACGTTGGATGGTCCGTGGCGCCAGCTTTG } \\
\text { ACGTTGGATGAGGAGGCCTCGTACTCTC } \\
\text { ACGTTGGATGTGGGGTCTTATCAAACCAC } \\
\text { ACGTTGGATGTAACCTACAAACCCTTCTGG } \\
\text { ACGTTGGATGTGGTGGTCTTGTCGTCAGG } \\
\text { ACGTTGGATGAGCTGAGGTCTGCCTTGATG }\end{array}$ & $\begin{array}{l}\text { ACGTTGGATGTATTTACAGGCTGGCTCAGG } \\
\text { ACGTTGGATGCTGCCAGAACACTATTCAAC } \\
\text { ACGTTGGATGCACTGCAGCTCAGATGTCTC } \\
\text { ACGTTGGATGTTTTCTGTGTCCTTCCCCTG } \\
\text { ACGTTGGATGAGATGTATCTGGTCAACTCC } \\
\text { ACGTTGGATGTTGGGTCTGGCTCTGTCTTC } \\
\text { ACGTTGGATGCGCATCTACACCTACATCGG } \\
\text { ACGTTGGATGGTTCTAATTTTTCTGGCTG } \\
\text { ACGTTGGATGTGGAGGCGCCGCGGGGCTCA } \\
\text { ACGTTGGATGAAGGAGCTTTATTGCTGCGG } \\
\text { ACGTTGGATGGTCTTGATTTAATCTCTAAGC } \\
\text { ACGTTGGATGACATCGCACATGGCAATTCC } \\
\text { ACGTTGGATGAGATCTACTGGGACTGCAAC } \\
\text { ACGTTGGATGACCACATACATCATTGCAAG }\end{array}$ & $\begin{array}{l}\text { GTGGTATCTGTAGGACG } \\
\text { tcCAATTCTTCCCCAGTTAATGATG } \\
\text { TTGTCTTGAAGTGGCTTTA } \\
\text { GCCTAGGCAGGAAACAGCAGAG } \\
\text { aaTCTTGTACTCTCATCTTACCTC } \\
\text { AGGAGTGTGTGTTGC } \\
\text { aTCCTGGTAGGGGTTCA } \\
\text { TGTTGCAGTCAGCAGATCACC } \\
\text { CCAGCTTTGGCTCTTT } \\
\text { gCGTACTCTCCGACCGC } \\
\text { GGTCTTATCAAACCACATAAGAGA } \\
\text { cCCTGGGCCAACAAGCTTGAGTGCGATC } \\
\text { gCAAGGCGACGGAACTGTATTTGGGA } \\
\text { CTTGATGGTAAAGTTAATACAGATTTT }\end{array}$ \\
\hline 2 & $\begin{array}{l}\text { ACC-4/5 } \\
\text { CD31 } \\
\text { CTL7A7 } \\
\text { DPH1 } \\
\text { HA-3 } \\
\text { HB-1 } \\
\text { HEATR1 } \\
\text { LB-PDK2B }\end{array}$ & $\begin{array}{l}\text { rs } 2289702 \\
\text { rs } 668 \\
\text { rs5758511 } \\
\text { rs35394823 } \\
\text { rs7162168 } \\
\text { rs } 161557 \\
\text { rs2275687 } \\
\text { rs751019 }\end{array}$ & $\begin{array}{l}\text { ACGTTGGATGACCGCAGACGGGGACTCCCA } \\
\text { ACGTTGGATGGCTCAGTTCCAAGGACTCAC } \\
\text { ACGTTGGATGTTGAGCACACCAGGCAAGTC } \\
\text { ACGTTGGATGTGCTGCTCTCTGAGATCTTC } \\
\text { ACGTTGGATGATGATGATGGGGCCCCAGC } \\
\text { ACGTTGGATGCTCAAGTCTCAGCTAAGCCA } \\
\text { ACGTTGGATGCTTCCTTTTTGATACCCAGC } \\
\text { ACGTTGGATGTGTTTCTTCCTCTGCAGGAC }\end{array}$ & $\begin{array}{l}\text { ACGTTGGATGATGTGGGCCACGCTGCCGCT } \\
\text { ACGTTGGATGGTACTGTGATTGTGAACAAC } \\
\text { ACGTTGGATGACGGAGATACCTCGTGGAAG } \\
\text { ACGTTGGATGATAGCCAGGCAGATACTCAC } \\
\text { ACGTTGGATGTAGAGAGGGAGTGCTCCTTT } \\
\text { ACGTTGGATGCTTCAACTTCAACCAATTCC } \\
\text { ACGTTGGATGTGGTTACCTGATCCACCAGA } \\
\text { ACGTTGGATGTCTCCTGGCAACTCACCAAT }\end{array}$ & $\begin{array}{l}\text { TCCCAGGAGCCAGGCCC } \\
\text { CACCTTCCACCAACA } \\
\text { CACACCAGGCAAGTCCCACACTC } \\
\text { CCCAGCAAGCTTAGC } \\
\text { cCTGGTGTGAGGGAAGTCA } \\
\text { CCATTCTTTTCTATAGGTTCTCTG } \\
\text { TTTATAAGTAAAGAGAGAGCAG } \\
\text { CCCCATGGTTTATATGAATGATA }\end{array}$ \\
\hline 3 & CD31 & rs 12953 & ACGTTGGATGGGCTGTGCAGTAATACTCTC & ACGTTGGATGAATGCCACCCAGGCATTTTG & CCCTCCTGTTCCTTG \\
\hline
\end{tabular}


identified as a potential new method for mHAgs genotyping. ${ }^{36,37}$ From a technical point of view, one of the main advantages of SNPs genotyping by MS system consists in the direct measurement of the mass of the molecules of interest without using any surrogate, such as fluorescence. MS genotyping has shown high accuracy; moreover, this methodology is rapid and highly automated, with a genotyping throughput of up to 128 matched pairs (256 samples) per run. The MS approach presents other advantages: it requires only a small amount of DNA, it is highly reproducible, and, furthermore, it works on multiplex and the design of each multiplex is made directly by the instrument software. The only drawbacks of this method are that it does not allow the genotyping of mHAgs resulting from deletions and can be used only if both the polymorphism and the polymorphism's flanking region are known. ${ }^{36}$ The use of designed primers for SNPs of interest and the MS protocol in this training set allowed us to genotype $100 \%$ of the SNPs (2116 genotypes of a predicted total number of 2116) and mHAgs. Intra- and extra-run controls demonstrated the reliability of this method. Analyzing the data obtained by genotyping the mHAgs of this set of donor/recipient pairs with their clinical features, particularly GvHD development and RFS, some interesting suggestions have emerged.

Sibling pairs have fewer mHAgs disparities despite the pairs with HLAmatched unrelated donor $(\mathrm{P}<0.0001)$. This data may appear obvious, but from a biological point of view no study has clearly shown that until now. This means that the genomic compatibility of HLA full matched MUD pairs will never be greater than full HLA sibling pairs.

Established that HLA differences between donor and recipient are the major predictor of GvHD, we investigated a possible role of mHAgs on GvHD development and relapse incidence in a training set of Ph-positive CML and ALL allotransplanted patients. These patients were chosen because representative of chronic and acute leukemias sharing a unique cytogenetic alteration: $t(9 ; 22)$. The only observation is that genomic DPH1 mismatch appeared to be related to an increased risk of grade $\geq 2$ aGvHD development. This possible correlation between DPH1 and aGvHD is supported by the fact that DPH1 is expressed by a broad range of non-hematopoietic tissues. The role of DPH1 on extramedullary toxicity has already been described by Warren, who pointed out that pulmonary toxicity was observed with infusion of DPH1-specific T

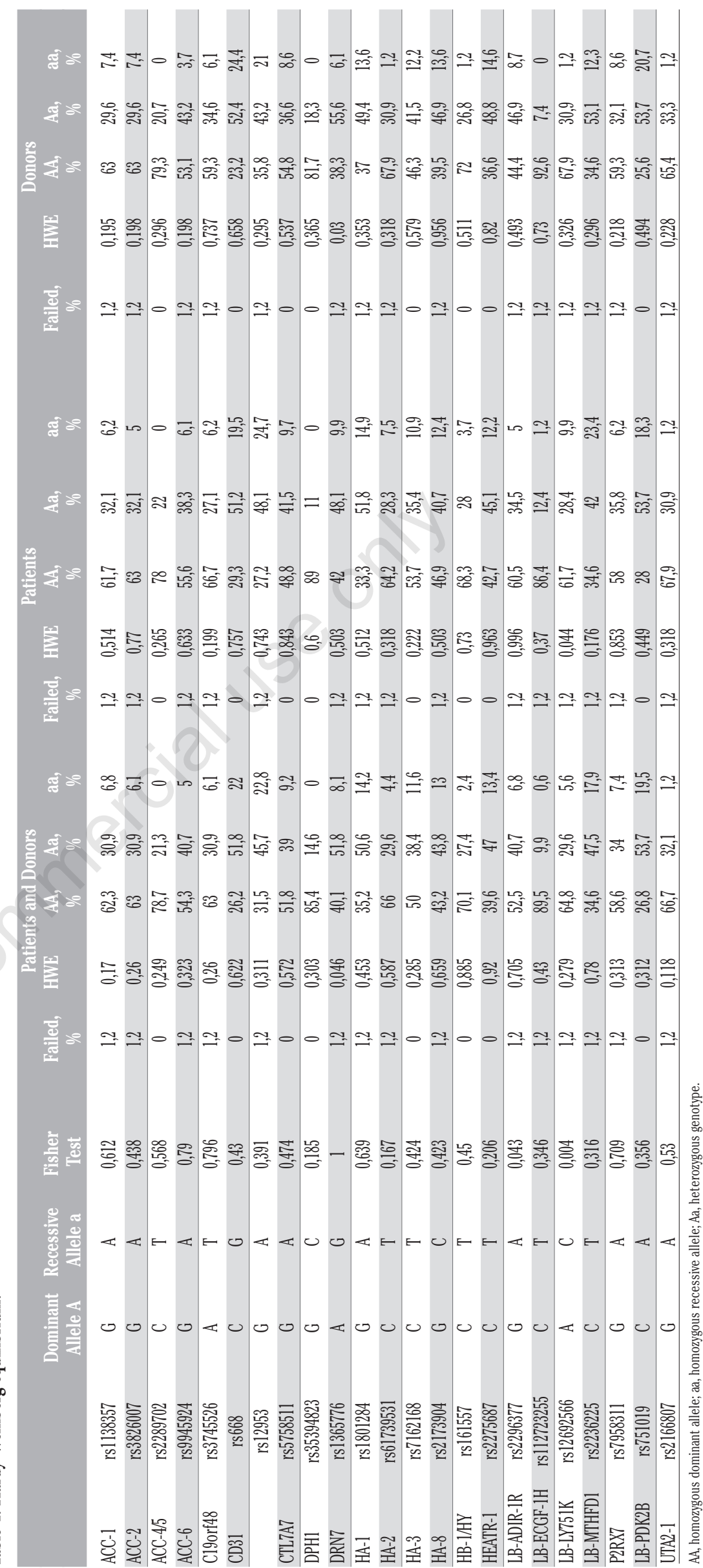


Table 5. Multivariate analysis of relationship btween mHAgs and aGvHD, cGvHD or RFS.

\begin{tabular}{|c|c|c|c|}
\hline ( & Grade $\geq 2$ aGvHD, HR (p) & cGvHD, HR (p) & RFS, HR (p) \\
\hline ACC-1 & ns & ns & ns \\
\hline ACC-2 & ns & ns & ns \\
\hline ACC-4 & ns & ns & ns \\
\hline ACC-5 & ns & ns & ns \\
\hline ACC-6 & ns & ns & ns \\
\hline C19orf48 & ns & ns & ns \\
\hline CD31 & ns & ns & ns \\
\hline CTL7A7 & ns & ns & ns \\
\hline DRN7 & ns & ns & ns \\
\hline DPH1 & $2.2(0.04)$ genomic mismatch & ns & ns \\
\hline HA-1 & ns & ns & ns \\
\hline HA-2 & ns & ns & ns \\
\hline $\mathrm{HA}-3$ & ns & ns & ns \\
\hline HA-8 & ns & ns & ns \\
\hline HB-1 & ns & ns & ns \\
\hline HEATR1 & ns & ns & ns \\
\hline LB-ADIR-1R & ns & ns & $0.3(0.03)$ genomic mismatch \\
\hline LB-ECGF1 & ns & ns & ns \\
\hline LB-MTHFD1 & $\mathrm{ns}$ & $\mathrm{ns}$ & $\mathrm{ns}$ \\
\hline LB-PDK2B & ns & ns & ns \\
\hline P2RX7 & ns & ns & ns \\
\hline UTA2-1 & ns & ns & ns \\
\hline
\end{tabular}

aGvHD: acute graft versus host disease; cGvHD: chronic graft versus host disease; GvL: graft versus leukemia; RFS: relapse free survival, RFS has been considered as surrogate marker of GvL

cells. On the contrary, leukemic blasts were poorly recognized by DPH1-specific $\mathrm{T}$ cells. ${ }^{43}$

Conversely, we found that genomic mismatch of LB-ADIR-1R on graft versus host direction was related to a better RFS. Our findings on LB-ADIR-1R mismatch are consistent with previous data from van Bergen, showing that LB-ADIR-1R specific $\mathrm{T}$ cells perform wide-reaching antitumor activity with a limited recognition of nonactivated tissues. Indeed, LB-ADIR-1R specific $\mathrm{T}$ cell recognize cell lines from haematological tumours, while generally mesenchymal and biliary epithelial cells are recognized to be GvHD target tissues. ${ }^{14}$

\section{Conclusions}

This work prove that MS may be a simple, effective, and accurate method for mHAgs genotyping. The method requires a small amount of gDNA that can be easily extracted also from cryopreserved cells. Furthermore, MS is able to genotype all mHAgs in a single work session, thus saving a lot of time.

Data analysis of our patients training set lead us to say that despite the full major HLA match, the minor-HLA genomic and immunogenic compatibility between a patient and his unrelated donor is always lower compared to the genomic and immunogenic compatibility of a patient and his sibling donor. In fact, sibling pairs had a lesser number of mHAgs mismatches compared to MUD pairs $(\mathrm{P}=0.003)$. Of 23 mHAgs evaluated, only 2, DPH1 and LBADIR-1R, proved to be correlated with the GvHD and GvL effect respectively, and these results confirm the previous reports. Our study suggests that MS would be used and useful for mHAgs genotyping. A larger and prospective trial would be warranted to validate this method.

\section{References}

1. haematological diseases, solid tumours and immune disorders: current practice in Europe 2009. Bone Marrow Transplant 2010;45:219-34.

2. Fowler DH, Gress RE. Th2 and Tc2 cells in the regulation of $\mathrm{GvHD}, \mathrm{GvL}$, and graft rejection: considerations for the allogeneic transplantation therapy of leukemia and lymphoma. Leuk Lymphoma 2000;38:221-34.

3. Ljungman P, Bregni M, Brune M, et al. Allogeneic and autologous transplantation for Bleakley M, Riddell SR. Molecules and mechanisms of the graft-versusleukemia effect. Nat Rev Cancer 2004;4:371-80.
4. Teshima T, Reddy P, Zeiser R. Reprint of: acute graft-versus-host disease: novel biological insights. Biol Blood Marrow Transplant 2016;223:S3-8.

5. Fontaine P, Roy-Proulx G, Xnafo L, et al. Adoptive transfer of minor histocompatibility antigen-specific $\mathrm{T}$ lymphocytes eradicates leukemia cells without causing graft-versus-host disease. Nat Med 2001;7:789-94.

6. Feng X, Hui KM, Younes HM, et al. Targeting minor histocompatibility antigens in graft versus tumor or graft versus leukemia responses. Trends Immunol 2008;29:624-32.

7. Spellman S, Warden S, Haagenson M, et al. Effects of mismatching for minor histocompatibility antigens on clinical outcomes in HLA-matched, unrelated hematopoietic stem cell transplants. Biol Blood Marrow Transplantat 2009;15:85663.

8. Laurin D, Hannani D, Pernollet M, et al. Immunomonitoring of graft-versus-host minor histocompatibility antigen correlates with graft-versus-host disease and absence of relapse after graft. Transfusion 2010;50:418-28.

9. Spierings E, Kim YH, Hendriks M, et al. Multicenter analyses demonstrate significant clinical effects of minor histocompatibility antigens on GvHD and GvL after HLA-matched related and unrelated hematopoietic stem cell 
transplantation. Biol Blood Marrow Transplant 2013;19:1244-53.

10. Brickner AG. Mechanisms of minor histocompatibility antigen immunogenicity: the role of infinitesimal versus structurally profound polymorphisms. Immunol Res 2006;36:33-41.

11. Spaapen R, Mutis T. Targeting haematopoietic-specific minor histocompatibility antigens to distinguish graft-versus-tumor effects from graftversus-host disease. Best Pract Res Clin Haematol 2008;21:543-57.

12. Simpson E. Minor histocompatibility antigens. Immunol Lett 1991;29:9-14.

13. Dolstra H, Fredrix H, Maas F, et al. A human minor histocompatibility antigen specific for B cell Acute Lymphoblastic Leukemia. J Exp Med 1999;189:301-8.

14. Kirker B, Stevanovic S, Urbanek M, et al. Induction of HA-1 specific cytotoxic T-cell clones parallels the therapeutic effect of donor lymphocyte infusion. Br J Haematol 2002;117:935-9.

15. Akatsuka Y, Warren EH, Gooley TA, et al. Disparity for a newly identified minor histocompatibility antigen, HA-8, correlates with acute graft-versus-host disease after haematopoietic stem cell transplantation from an HLA-identical siblilng. Br J Haematol 2003;123:671-5.

16. Nishida T, Akatsuka Y, Morishima Y, et al. Clinical relevance of a newly identified HLA-A24-restricted minor histocompatibility antigen epitope derived from BCL2A1, ACC-1, in patients receiving HLA genotypically matched unrelated bone marrow transplant. Br J Haematol 2004;124:629-35.

17. Wilson W 3rd, Pardo-Manuel de Villena F, Lyn-Cook BD, et al. Characterization of a common deletion polymorphism of the UGT2B17 gene linked to UGT2B15. Genomics 2004;84:707-14.

18. Brickner AG, Evans AM, Mito JK, et al. The PANE1 gene encodes a novel human minor histocompatibility antigen that is selectively expressed in B-lymphoid cella and B-CLL. Blood 2006;107:3779-86.

19. Tykodi SS, Fujii N, Vigeron N, et al. C19orf48 encodes a minor histocompatibility antigen recognized by CD8+ cytotoxic $\mathrm{T}$ cells from renal cell carcinoma patients. Clin Cancer Res 2008;14:5260-9.

20. Stumpf AN, van der Meijden ED, van Bergen CA, et al. Identification of 4 new HLA-DR-restricted minor histocompatibility antigens as hematopoietic targets in antitumor immunity. Blood 2009; 114:3684-92.

21. Spierings E, Drabbels J, Hendriks M, et al. A uniform genomic minor histocompatibility antigen typing methodology and database designed to facilitate clinical applications. PLoS One 2006; 1:e42.

22. Goulmy E, Schipper R, Pool J, et al. Mismatches of minor histocompatibility antigens between HLA-identical donors and recipients and the development of graft-versus-host disease after bone marrow transplantation. N Engl J Med 1996;334:281-5.

23. Goulmy E. Human minor histocompatibility antigens. Curr Opin Immunol 1996;8:75-81.

24. Kircher B, Wolf B, Stevanovic S, et al. Hematopoietic lineage-restricted minor histocompatibility antigen HA-1 in graftversus-leukemia activity after donor lymphocyte infusion. J Immunother 2004;27:156-60.

25. Perez-Garcia A, De la Camára R, Torres A, et al. Minor histocompatibility antigen HA-8 mismatch and clinical outcome after HLA-identical sibling donor allogeneic stem cell transplantation. Haematologica 2005;90:1723-4.

26. Spierings E, Goulmy E. Expanding the immunotherapeutic potential of minor histocompatibility antigens. J Clin Invest 2005;115:3397-400.

27. Bertinetto FE, Dall'Olmo AM, Mazzola GA, et al. Role of non-HLA genetic polymorphysms in graft-versus-host disease after haematopoietic stem cell transplantation. Int $\mathrm{J}$ Immunogenet 2006;33:375-84.

28. Akatsuka Y, Morishima Y, Kuzushima K, et al. Minor histocompatibility antigens as targets for immunotherapy using allogenic immune reactions. Cancer Sci 2007;98:1139-46.

29. Maruya E, Saji H, Seki S, et al. Evidence that $\mathrm{CD} 31, \mathrm{CD} 49 \mathrm{~b}$, and $\mathrm{CD} 62 \mathrm{~L}$ are immunodominant minor histocompatibility antigens in HLA identical sibling bone marrow transplants. Blood 1998;92:2169-76.

30. Gallardo D, Aróstegui JI, Balas A, et al. Disparity for the minor histocompatibility antigen HA-1 is associated with an increased risk of acute graft-versus-host disease (GvHD) but it does not affect chronic GvHD incidence, disease-free survival or overall survival after allogeneic human leucocyte antigen-identical sibling donor transplantation. $\mathrm{Br} \mathrm{J}$ Haematol 2001;114:931-6.

31. Dickinson AM. Non-HLA genetics and predicting outcome in HSCT. Int $\mathrm{J}$ Immunogenet 2008;35:375-80.

32. Markiewicz M, Siekiera U, Karolczyk A, et al. Immunogenic disparities of 11 minor histocompatibility antigens (mHAs) in HLA-matched unrelated allogeneic hematopoietic SCT. Bone Marrow Transplant 2009;43:293-300.

33. Larsen ME, Kornblit B, Larsen MV, et al. Degree of predicted minor histocompatibility antigen mismatch correlates with poorer clinical outcomes in nonmyeloablative allogeneic hematopoietic cell transplantation. Biol Blood Marrow Transplant 2010;16:137081.

34. Olerup O, Zetterquist H. HLA-DR typing by PCR amplification with sequencespecific primers (PCR-SSP) in 2 hours: an alternative to serological DR typing in clinical practice including donor-recipient matching in cadaveric transplantation. Tissue Antigens 1992;39:225-35.

35. Dunbar SA. Applications of Luminex xMAP technology for rapid, highthroughput multiplexed nucleic acid detection. Clin Chim Acta 2006;363:7182.

36. Van Bergen CA, Rutten CE, Van Der Meijden ED, et al. High-throughput characterization of 10 new minor histocompatibility antigens by whole genome association scanning. Cancer Res 2010;70:9073-83.

37. Stanssens P, Zabeau M, Meersseman G, et al. High-throughput MALDI-TOF discovery of genomic sequence polymorphisms. Genome Res 2004; 14: 126-33.

38. Maresso K, Broeckel U. Genotyping platforms for mass-throughput genotyping with SNPs, including human genomewide scans. Adv Genet 2008;60:107-39.

39. Glucksberg H, Storb R, Fefer A, et al. Clinical manifestations of graft-versushost disease in human recipients of marrow from HL-A-matched sibling donors. Transplantation 1974;18:295-304.

40. Filipovich AH, Weisdorf D, Pavletic S, et al. National Institutes of Health consensus development project on criteria for clinical trials in chronic graft-versus-host disease: I. Diagnosis and staging working group report. Biol Blood Marrow Transplant 2005;11:945-56.

41. Jurinke C, van den Boom D, Cantor CR, et al. Automated genotyping using the DNA MassArray technology. Methods Mol Biol 2002;187:179-92.

42. Kaplan ER, Mejer P. Non parametric estimation from incomplete observation. J Am Stat Assoc 1958;53:457-81.

43. Warren EH, Fujii N, Akatsuka Y, et al. Therapy of relapsed leukemia after allogeneic hematopoietic cell transplantation with $\mathrm{T}$ cells specific for minor histocompatibility antigens. Blood 2010;115:3869-78. 